Textures and Microstructures, 1991, Vols 14-18, pp. 1007-1012 Reprints available from the publisher

Photocopying permitted by license only (c) 1991 Gordon and Breach Science Publishers SA Printed in the United Kingdom

\title{
ANISOTROPIC FINITE-ELEMENT ANALYSIS OF PLASTIC METALFORMING PROCESSES
}

\author{
A. VAN BAEL ${ }^{\star}$, P. VAN HOUTTE ${ }^{\star}$, E. AERNOUDT ${ }^{\star}$, \\ F.R. HALL ${ }^{\star \star}$, I. PILLINGER ${ }^{\star \star}$, P. HARTLEY ${ }^{\star \star}$, C.E.N. STURGESS ${ }^{\star \star}$ \\ ${ }^{\star}$ Dept. of Metallurgy and Materials Engineering, K.U. Leuven, Belgium \\ ${ }^{\star \star}$ School of Automotive Engineering, University of Birmingham, U.K.
}

\section{ABSTRACT}

This paper describes some aspects of the incorporation of plastic anisotropy of textured materials into an elastic-plastic finite-element program in order to simulate the deformation of such materials.

\section{INTRODUCTION}

Finite-element techniques have proved to be very valuable tools for the prediction of material flow and stress distributions in a wide variety of metalforming processes. A very important aspect of the finite-element formulation is the model that is used to describe the material behaviour. This may be clear from the fact that the material flow during a forming process is not only governed by the geometry of the forming tools, but may be strongly influenced by material properties such as work hardening and plastic anisotropy.

Most of the existing finite-element programs assume that the material is isotropic, and some programs make an allowance for the fact that the plastic properties may be anisotropic, usually by using Hill's anisotropic yield behaviour. While Hill's model is well suited for specific metals and textures, it is insufficient for a proper description of the plastic behaviour of most anisotropic materials. Therefore a very general mathematical model for plastic anisotropy has been developed, and this model is presently being incorporated into a previously existing elastic-plastic finite-element program for the simulation of forming processes.

\section{ANISOTROPIC PLASTIC MODEL}

The formulation that is used here to describe the anisotropic plastic material behaviour is the series expansion method explained elsewhere [1] by Van Houtte et al. This method is based on the existence of a plastic potential $Q$, termed a plastic potential of type II in [1], which is a function of the plastic strain rate, $\dot{\varepsilon}_{i j}^{p l}$, of the following form :

$$
Q\left(\dot{\varepsilon}_{i j}^{p l}\right)=\left\|\dot{\varepsilon}^{p l}\right\| F\left(\frac{\dot{\varepsilon}_{i j}^{p l}}{\left\|\dot{\varepsilon}^{p l}\right\|}\right)
$$

where $\left\|\dot{\varepsilon}^{p l}\right\|$ is the magnitude of the plastic strain rate tensor $\dot{\varepsilon}^{p l}$, and $F$ is a function of the so-called plastic strain rate mode. The term series expansion 
method refers to the fact that at present series expansions are being used as a particular mathematical representation of the function $Q$. Series expansions with even order terms up to sixth order are used at present, containing 296 coefficients. The values of these coefficients have to be determined first before the model can be used to represent the plastic behaviour of a given material.

In [1] it is explained how these coefficients can be obtained for a polycrystalline material. In this approach it is assumed that plastic anisotropy is completely determined by the crystallographic texture of the material. The information about this texture has to be obtained first in the form of the crystallographic orientation distribution function (C.O.D.F). Once the crystal structure (FCC, BCC , ...) and the C.O.D.F. of the material are known, a Taylor-Bishop-Hill model for polycrystal plasticity is used to perform a large number of simulations (e.g. 9500) each for a different plastic deformation $\dot{\varepsilon}_{i j}^{p l}$ of the polycrystal. A well-known result of each of these calculations is the so-called average polycrystal Taylor factor which corresponds to the particular plastic deformation $\dot{\varepsilon}_{i j}^{p l}$. In [1] it is demonstrated that the coefficients of the series expansion approximation of $F$ can be obtained by a least squares fitting of the series expansion to the large number of average polycrystal Taylor factors. Software developed so far allows a very fast calculation of the values of the series expansion coefficients of $F$ for polycrystalline materials with FCC or BCC crystal structure.

It is remarked here that the potential function of type II determines the shape of the yield locus, but not its size. In the context of traditional TaylorBishop-Hill models the scaling factor can be identified as the critical resolved shear stress $\tau_{c}$. Strain hardening effects can be incorporated into the present formulation by using a scaling factor $\tau_{c}$ which is a function of $\gamma$, with $\gamma$ being a scalar measure for the accumulated plastic strain. Information about the function $\tau_{c}(\gamma)$ of a given material has to be obtained from mechanical tests such as tension, compression or torsion tests.

\section{ANISOTROPIC ELASTIC-PLASTIC CONSTITUTIVE FE-MODEL}

A detailed discussion of the finite-element (FE) theory is given in [2]. In the work described here an incremental approach is adopted, which means that a deformation process is simulated in a number of consecutive deformation increments. Furthermore the deforming body is partitioned into a number of elemental regions (i.e. finite elements), and the material behaviour of each element during an increment is approximated by a linearized relation of the following form :

$$
\Delta \underline{\sigma}=[\mathrm{D}] \Delta \underline{\varepsilon}
$$

in which $\Delta \underline{\sigma}$ is a stress increment and $\Delta \underline{\varepsilon}$ is the corresponding strain increment, both expressed in 6-dimensional column matrix representations. The FEformulation that is used here is completely elastic-plastic. This means that elastic deformations are not neglected where plastic flow occurs and that parts of the deforming body may be deforming completely elastic. The matrix [D] will be different for elements which are deforming elastically and for elements where plastic flow occurs. In the isotropic FE-program equation (2) represents Hooke's law for elastically deforming elements, and the Lévy-Mises relations for plastically deforming elements. In the anisotropic version of the FE-program the following expression is used for elastically deforming elements : 


$$
\Delta \underline{\sigma}=\left[\mathrm{D}^{e l}\right] \Delta \underline{\varepsilon}
$$

and the following for plastically deforming elements :

$$
\Delta \underline{\sigma}=\left(\left[\mathrm{D}^{e l}\right]-\left[\mathrm{D}^{p l}\right]\right) \Delta \underline{\varepsilon}
$$

where :

$$
\left[\mathrm{D}^{p l}\right]=\frac{\left[\mathrm{D}^{e l}\right] \dot{\varepsilon}^{p l_{o}}\left(\left[\mathrm{D}^{e l}\right] \dot{\varepsilon}^{p l_{o}}\right)^{\top}}{\left(\left[\mathrm{D}^{e l}\right] \dot{\varepsilon}^{p l_{o}}\right)^{\top} \dot{\varepsilon}^{p l_{o}}+\left(\frac{\mathrm{d} \tau_{c}}{\mathrm{~d} \gamma}\right)^{o}\left(F^{o}\left\|\dot{\varepsilon}^{p l_{o}}\right\|\right)^{2}}
$$

$\dot{\varepsilon}^{p l_{0}}$ is the plastic strain rate at the start of the increment and $\left\|\dot{\varepsilon}^{p l_{o}}\right\|$ is its magnitude; $\left(\frac{\mathrm{d} \tau_{c}}{\mathrm{~d} \gamma}\right)^{o}$ is the derivative of the critical resolved shear stress $\tau_{c}$ with respect to $\gamma$ at the start of the increment, and $F^{\circ}$ is the value of the series expansion at the start of the increment. Equations (4) and (5) which have been derived by Pillinger et al. in [3] allow for both elastic and plastic anisotropy.

\section{CALCULATING CORRECT STRESS INCREMENTS}

The constitutive relations (3) and (4) between stress increments $\Delta \underline{\sigma}$ and strain increments $\Delta \varepsilon$ are used by the FE program to construct the so-called global stiffness equation of the deforming body. After solving this stiffness equation the deformations throughout the body are known, and the strain increments $\Delta \underline{\varepsilon}$ can be calculated in all elements. At this point the constitutive relations between stress and strain increments could be used again, this time to calculate the stress increments $\Delta \underline{\sigma}$. The resulting stresses $\underline{\sigma}$ could then be found by adding the stress increments $\Delta \underline{\sigma}$ to the stresses at the start of the deformation increment, $\underline{\sigma}^{o}$. The stresses $\underline{\sigma}$ found in this way however will in general not satisfy the yield condition. This means that these stresses will very often lie outside the yield locus, a fact which is physically impossible. A different strategy has therefore been developed to calculate stress increments corresponding to known strain increments.

First it is assumed that the deformation $\Delta \underline{\varepsilon}$ is completely elastic. Equation (3) is therefore used to calculate the so-called hypothetical-elastic stress increment $\Delta \underline{\sigma}^{e}$, and from this the so-called hypothetical-elastic stress $\underline{\sigma}^{e}$ is calculated by $\underline{\sigma}^{e}=\underline{\sigma}^{o}+\Delta \underline{\sigma}^{e}$. It is then checked whether $\underline{\sigma}^{e}$ lies inside the anisotropic yield locus. Figure 1 shows two typical situations. With reference to Figure 1(a), if the deformation is elastic at the start of the increment having stress-state $\underline{\sigma}^{\circ}$ and if at the end of the increment $\underline{\sigma}^{e}$ lies inside the estimated yield locus, then the correct stress $\underline{\sigma}$ at the end of the deformation step will be equal to the stress $\underline{\sigma}^{e}$. Referring to Figure 1(b), if the deformation is elastic at the start of the increment and if at the end of the increment $\underline{\sigma}^{e}$ lies outside the estimated yield locus, then the stress-path between $\underline{\sigma}^{\circ}$ and $\underline{\sigma}^{e}$ must cross the yield locus at a certain fraction $r$ of the deformation step. An iterative procedure has been developed for anisotropic yield loci to calculate this totally elastic fraction $r$ and the stress-state $\underline{\sigma}^{r}$ which defines the point at which plastic flow initiates during the deformation increment. 

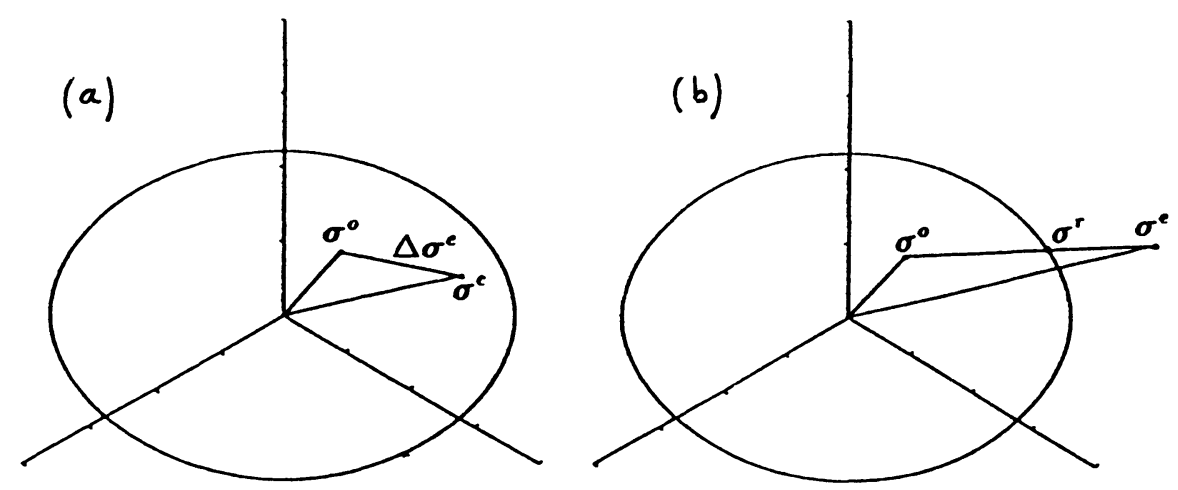

Figure 1. Stress-states in (a) elastically deforming element, and (b) where plastic flow initiates.

From that point onwards the stress-states should remain on the yield locus. In the isotropic version of the FE-program it is ensured that the stress-state at the end of such an increment lies exactly on the von Mises yield surface, which is accomplished using the so-called mean normal technique described by Pillinger et al. in [4]. For the anisotropic version an analogous iterative procedure has been developed of which the basic assumptions are summarized here :

1. The total strain increment during plastic flow, $\Delta \underline{\varepsilon}$, consists of an elastic part $\Delta \underline{\varepsilon}^{e l}$ and a plastic part $\Delta \underline{\varepsilon}^{p l}$. This is expressed by :

$$
\Delta \underline{\varepsilon}=\Delta \underline{\varepsilon}^{e l}+\Delta \underline{\varepsilon}^{p l}
$$

2. The relation between the stress increment, $\Delta \underline{\sigma}$, and the elastic part of the strain increment during plastic flow, $\Delta \underline{\varepsilon}^{e l}$, is given by (cf. equation (3)) :

$$
\Delta \underline{\sigma}=\left[\mathrm{D}^{e l}\right] \Delta \underline{\varepsilon}^{e l}
$$

3. From equations (6) and (7) it follows that :

$$
\Delta \underline{\sigma}=\left[\mathrm{D}^{e l}\right] \Delta \underline{\varepsilon}-\left[\mathrm{D}^{e l}\right] \Delta \underline{\varepsilon}^{p l}
$$

In this equation the plastic strain increment $\Delta \underline{\varepsilon}^{p l}$ is not known. It is assumed that the plastic strain rate mode during the increment, $\frac{\dot{\varepsilon}^{p l}}{\left\|\dot{\varepsilon}^{p l}\right\|}$, is constant. The plastic strain increment $\Delta \varepsilon^{p l}$ is then given by :

$$
\Delta \underline{\varepsilon}^{p l}=m \frac{\underline{\dot{\varepsilon}}^{p l}}{\left\|\dot{\varepsilon}^{p l}\right\|}
$$

where $m$ is a proportionality factor.

From equations (8) and (9) it follows that :

$$
\Delta \underline{\sigma}=\left[\mathrm{D}^{e l}\right] \Delta \underline{\varepsilon}-m\left[\mathrm{D}^{e l}\right] \frac{\dot{\varepsilon}^{p l}}{\left\|\dot{\varepsilon}^{p l}\right\|}
$$

The factor $m$ is then determined iteratively in such a way that the stressstate $\underline{\sigma}$ defined by $\underline{\sigma}=\underline{\sigma}^{\circ}+\Delta \underline{\sigma}$ lies on the anisotropic yield locus. It is remarked here that a sensible choice for the constant plastic strain rate mode must be made in order to obtain accurate stress predictions. 


\section{SINGLE ELEMENT TESTING OF THE FE-PROGRAM}

The incorporation of anisotropy into the FE-program involves modifications to several aspects of the program, and simple benchmark tests have proved invaluable to verify these changes. A very useful test is the NAFEMS single-element benchmark for triaxial displacement [5]. In this test a single cubic element undergoes 12 prescribed displacement steps as shown in Figure 2. The first 6 displacement steps are tensile and the following 6 are compressive returning the element back to its original cubic shape. The interesting feature of this benchmark is that the deformation pattern is completely imposed on the deforming body. Therefore this benchmark is especially suited to verify the section of the FE-program in which stresses corresponding to known strain increments are calculated.
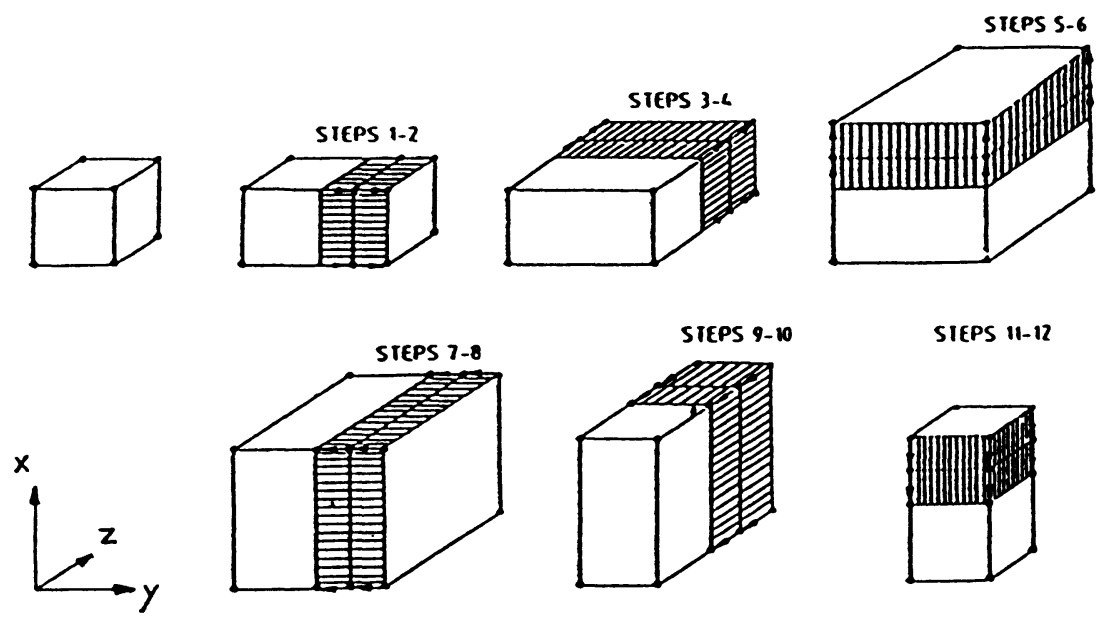

SIEPS $11-12$

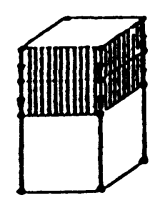

Figure 2. Prescribed displacement history for triaxial benchmark test.
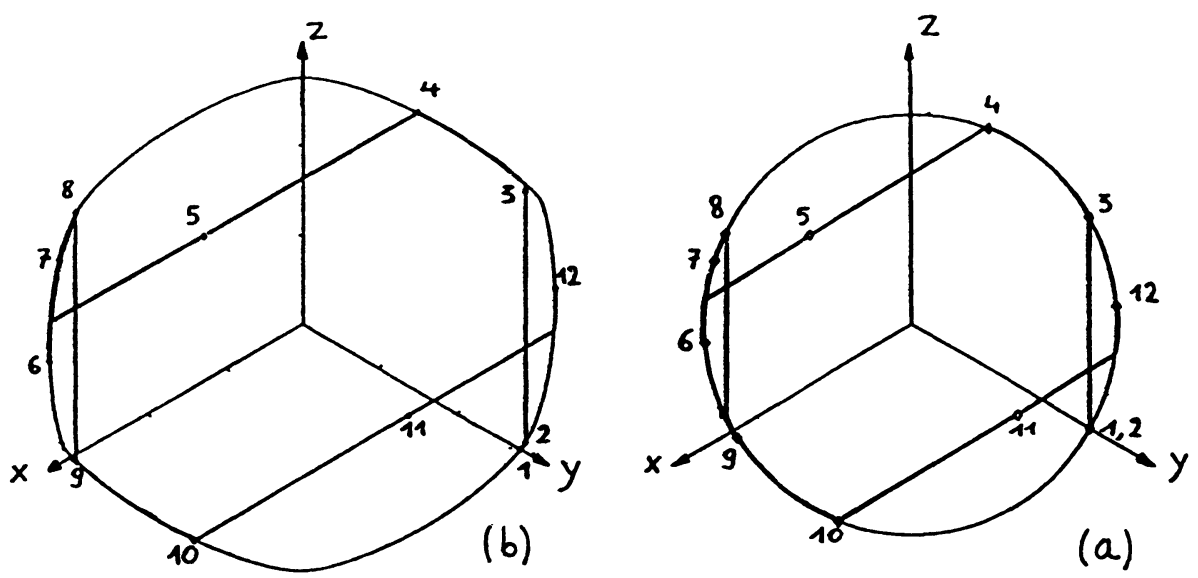

Figure 3. Stress-path during triaxial benchmark test for (a) isotropic and (b) anisotropic material. 
The NAFEMS benchmark has been performed with the anisotropic version of the FE-program for materials with different yield loci. Results of two simulations are presented here. An initial test has been performed on the basis that the series expansion method for yield loci can be used to predict the same plastic behaviour as is predicted by the isotropic FE-program (i.e. the von Mises yield criterion and the associated Lévy-Mises flow rule). This will be the case when a series expansion $F$ with only a term of zero order is used. Therefore, for this special case, the same FE results should be obtained with the anisotropic version of EPFEP3 as with the original isotropic FE-program. This has been verified successfully and the results are shown in Figure 3(a). In this figure the stress-path followed by the element during the 12 displacements is represented in a pi plane section of the scaled yield locus in stress-space. A second test is performed for a low carbon steel of which the anisotropic yield locus has been obtained from the C.O.D.F. of the material. The results are represented in Figure 3(b), clearly showing the anisotropic nature of the yield locus and the stress-path correctly connecting with it.

\section{FUTURE WORK}

The anisotropic FE-program is being developed in order to study plastic forming processes in which material anisotropy is very important, such as in several sheet forming operations. Before the FE-program can be successfully applied for such complex simulations it is necessary to further verify the validity of the anisotropic FE-formulation. Strain hardening for instance is one of the aspects which has not been implemented fully yet, allthough the anisotropic FEformulation allows for such material behaviour. Furthermore the FE-program will be tested for simulations in which the material flow is not completely imposed as in the NAFEMS benchmark, such as the simulation of a uniaxial tensile test of sheet material. The FE-program should be able to predict the anisotropic material flow which is experimentally observed in the form of the well-known r-factor.

\section{ACKNOWLEDGEMENT}

This work has been carried out as part of the Brite/Euram project BREU^ 0107-c funded by the Commission of the European Communities.

\section{REFERENCES}

1. P. Van Houtte, K. Mols, A. Van Bael and E. Aernoudt, Textures and Microstructures, 11 (1989) 23-29

2. G.W. Rowe, C.E.N. Sturgess, P. Hartley and I. Pillinger, Finite-element plasticity and metalforming analysis, Cambridge: CUP (1990)

3. I. Pillinger, P. Hartley, C.E.N. Sturgess, A. Van Bael, P. Van Houtte and E. Aernoudt, Finite-element analysis of anisotropic material deformation, Suppl. Proc. 3rd Int. Conf. on Numerical Methods in Industrial Forming Processes, Fort Collins, Colorado, 26-30 June 1989, E.G. Thompson, R.D. Wood, O.C. Zienkiewicz and A. Samuelsson, Eds., A.A. Balkema, Rotterdam, (1989) 6 pages

4. I. Pillinger, P. Hartley, C.E.N. Sturgess and G.W. Rowe, Int. J. Mech. Sci., Vol. 28, No. 1 (1986) 23-29

5. E. Hinton, M. H. Ezatt, Fundamental Tests for Two and Three Dimensional, Small Strain, Elastoplastic Finite Element Analysis (1987) 